\title{
Pretreatment tumor standardized uptake value as a prognostic factor in primary head and neck squamous cell carcinoma
}

\author{
BAITAO ZHANG ${ }^{1}$, FANG NIE $^{2}$, BIN JIN $^{1}$, QINGHONG MENG ${ }^{1}$ and PIN DONG ${ }^{1}$ \\ ${ }^{1}$ Department of Otolaryngology-Head and Neck Surgery, Shanghai First People's Hospital, Shanghai Jiao Tong University, \\ Shanghai 200080; ${ }^{2}$ Department of Intensive Care Medicine, Ren Ji Hospital, School of Medicine, \\ Shanghai Jiao Tong University, Shanghai 200001, P.R. China
}

Received August 21, 2014; Accepted October 9, 2014

DOI: $10.3892 / \mathrm{mco} .2014 .464$

\begin{abstract}
Our objective was to conduct a systematic review and meta-analysis of studies assessing the diagnostic performance of primary tumor standardized uptake value (SUV) in head and neck squamous cell carcinoma (HNSCC), as measured by positron emission tomography. A systematic search of the indexed medical literature was conducted using appropriate keywords to identify relevant studies. Six articles were identified by searching electronic databases. A statistical analysis was performed with RevMan 4.2.2 software. SUV measurement and the SUV threshold for defining high SUV were studied dependently. For each publication, we first obtained an estimate of the relative risk (RR) for comparing patients with a low and those with a high SUV. Subsequently, we aggregated the individual RRs into a combined RR using the fixed-effects model to yield weighted mean pooled estimates with $95 \%$ confidence intervals (CIs). Publication bias was assessed with a funnel plot. A total of 6 clinical trials involving 453 patients were included in the meta-analysis. The combined RR from the 6 reports for local control was 0.72 (95\% CI: 0.63-0.81). The funnel plot revealed symmetrical distribution, indicating no evidence of significant publication bias. The increase in the SUV of the primary tumor was found to be a poor prognostic marker for patients with HNSCC in the meta-analysis, which requires further confirmation in a meta-analysis based on individual patient data.
\end{abstract}

Correspondence to: Professor Pin Dong, Department of Otolaryngology-Head and Neck Surgery, Shanghai First People's Hospital, Shanghai Jiao Tong University, 100 Haining Road, Shanghai 200080, P.R. China

E-mail: dongpin64@aliyun.com

Key words: standardized uptake value, meta-analysis, head and neck squamous cell carcinoma, positron emission tomography, local control, primary tumor

\section{Introduction}

With the exception of stage, no definitive prognostic factors have been established for head and neck cancer. Cancer stage is currently the most important prognostic factor for outcome. Traditional staging of head and neck squamous cell carcinoma (HNSCC) depends on the site of disease origin, size and extent of the primary tumor, cervical lymph node involvement and presence or absence of distant metastasis. This staging system relies on conventional imaging, such as computed tomography (CT) and magnetic resonance imaging (MRI); however, this approach is limited by outcome heterogeneity within stage categories, hampering accurate prognostication for individual patients (1).

Clinical characteristics, including gender, age, weight loss and biological markers, such as p53 (2), cyclin D (3) and epidermal growth factor receptor (4), have been investigated but are not sufficiently accurate for individual patient prognosis. More accurate markers would be helpful to stratify patients for therapy and predict outcomes.

As with other types of cancer, there is great interest in trying to identify factors predictive of outcome other than stage and biological markers. The development of functional imaging studies, particularly positron emission tomography (PET) with the glucose analogue $2-\left({ }^{18} \mathrm{~F}\right)$-fluoro-2-de oxy-D-glucose $\left({ }^{18} \mathrm{~F}-\mathrm{FDG}\right)$ has emerged as a useful tool for several malignancies. PET scanning enables non-invasive study of the physiology of cancers (5). Tumor uptake of ${ }^{18} \mathrm{~F}-\mathrm{FDG}$, standardized uptake value (SUV), as measured by PET, has been associated with various cellular characteristics, such as cell viability (6) and proliferative activity (7). Furthermore, SUV as a semi-quantitative simplified measurement of tissue deoxyglucose metabolic rate has suggested that tumor FDG uptake may be of prognostic significance, as patients with high FDG uptake generally have a less favorable outcome (8).

Other retrospective studies have investigated the prognostic significance of SUV, but the majority of those reports only included a small patient sample. Based on these considerations, we performed a systematic review of the literature on ${ }^{18}$ F-FDG-PET scan and local control and a meta-analysis of the data to determine the prognostic value of primary tumor SUV in patients with head and neck cancer. 
Table I. Characteristics of studies included in this meta-analysis.

\begin{tabular}{lcccc}
\hline Author (year) & $\begin{array}{c}\text { Total } \\
\text { patient no. }\end{array}$ & $\begin{array}{c}\text { SUV<threshold } \\
\text { patient no. }\end{array}$ & $\begin{array}{c}\text { SUV>threshold } \\
\text { patient no. }\end{array}$ & Histology \\
\hline Brun et al $(2002)$ & 44 & 23 & 21 & HNSCC \\
Allal et al $(2004)^{\mathrm{a}}$ & 119 & 62 & 57 & HNSCC \\
Kim et al $(2007)$ & 52 & 27 & 25 & HNSCC \\
Roh et al $(2007)$ & 79 & 48 & 31 & HNSCC \\
Liao et al $(2009)$ & 109 & 97 & 12 & HNSCC \\
Torizuka et al (2009) & 50 & 21 & 29 & $(12)$ \\
\hline
\end{tabular}

ancluded 3 patients with unknown primary tumors and 2 with T1-2 tumors; in these 5 patients, the SUV of the lymph node was used as reference. SUV, standardized uptake value; HNSCC, head and neck squamous cell carcinoma.

Table II. Main SUV characteristics reported in the six publications assessable for meta-analysis.

\begin{tabular}{|c|c|c|c|c|c|}
\hline Study (year) & Type of SUV & Correction of SUV & SUV threshold definition & SUV threshold & (Refs.) \\
\hline Brun et al (2002) & $\mathrm{SUV}_{\text {mean }}$ & Weight & Median & 9.0 & (10) \\
\hline Allal et al (2004) $)^{\mathrm{a}}$ & $\mathrm{SUV}_{\max }$ & Weight & Median & 4.76 & (11) \\
\hline Kim et al (2007) & $\mathrm{SUV}_{\max }$ & Lean body weight & Median & 6.0 & (12) \\
\hline Roh et al (2007) & $\mathrm{SUV}_{\max }$ & Weight & Best cut-off ${ }^{b}$ & 8.0 & (13) \\
\hline Liao et al (2009) & $\mathrm{SUV}_{\text {max }}$ & Weight & Best cut-off ${ }^{b}$ & 19.3 & (14) \\
\hline Torizuka et al (2009) & $\mathrm{SUV}_{\max }$ & Weight & Best cut-off ${ }^{b}$ & 7.0 & (15) \\
\hline
\end{tabular}

ancluded 3 patients with unknown primary tumors and 2 with T1-2 tumors; in these 5 patients, the SUV of the lymph node was used as reference. ${ }^{b}$ The authors maximized the log-rank test statistic to determine the optimal cut-off. SUV, standardized uptake value.

\section{Materials and methods}

Search strategy. We conducted a search, without language restrictions, through PubMed, Embase, the Cochrane Controlled Trials Register and OVID databases. We employed both medical subject headings and free-language terms for 'HNSCC' (i.e., 'head and neck cancer', 'head and neck carcinoma', 'squamous cell carcinoma'), combined with each of the following: "positron emission tomography or PET or PET imaging tomography' and 'FDG-F' ${ }^{18}$ or FDG or ${ }^{18} \mathrm{~F}$-fluorodeoxyglucose or ${ }^{18} \mathrm{~F}$-FDG' and 'SUV or standardized uptake value' as search terms. The references reported in all the identified studies were used to complete this search, which ended in May, 2014. Further searches were conducted by scanning the abstracts of major ENT and nuclear medicine meetings.

Inclusion and exclusion criteria. In order to be eligible for the systematic review, a study was required to fulfill the following criteria: i) Limited to HNSCC, with any stage or any histological grading; ii) assessed the association between pretherapeutic SUV and local control, at least in a univariate analysis; iii) SUV referred to the primary tumor; and iv) reports using all modalities of care were included.

Abstracts were excluded, as they do not provide sufficient details to assess methodology or relative information to perform a meta-analysis. Furthermore, we carefully examined the possibility of patient duplication by reporting the same cohorts in different publications. This led us to suppress one article, although no reference to such duplicates was reported by the authors.

Quality assessment. To improve quality assessment, 7 physicians and 1 biostatistician reviewed all the publications to assess their methodological quality and extract the most important information determining the clinical and PET characteristics. A methodological quality scale was designed for the purpose of this study, using the variables available in the publications (9). This score assessed the clinical and PET reports. The clinical report included the distribution of the expected 'prognostic factors' (age, gender, stage, performance status, histological grading and weight loss), tumor stage description, staging characteristics [definition of the size of pathological metastasis, systematic use of the head and neck CT for head and neck staging, systematic metastatic work-up, systematic use of a CT or MRI for distant metastasis, histological confirmation of metastasis and if the analysis of the association between SUV and each expected prognostic factor was performed without knowledge of clinical results and vice versa (double blind)], description of results of local control and analyses (number of patients, number of local control, follow-up duration, number of patients lost to follow-up, univariate and multivariate analyses, description of statistical tests, definition of local control and SUV cut-off definition). The PET report 


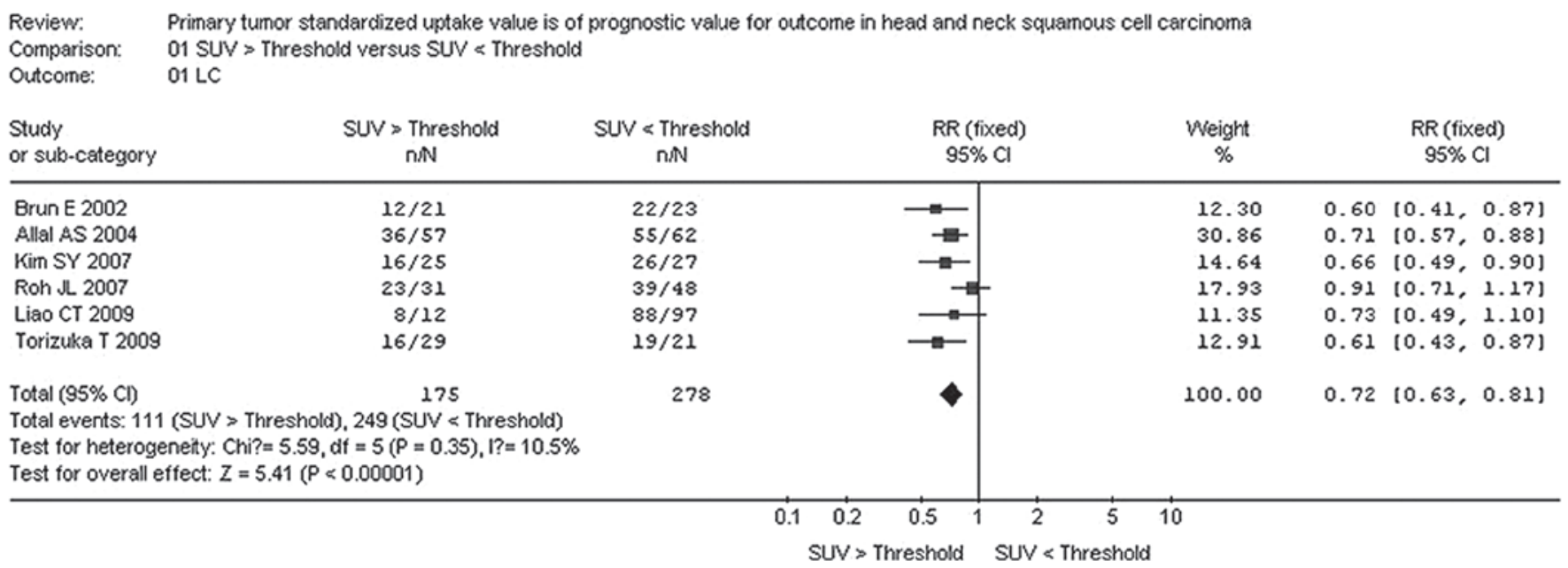

Figure 1. Graph of SUV>threshold vs. SUV<threshold on local control of patients with head and neck squamous cell carcinoma. SUV, standardized uptake value; RR, relative risk; $95 \%$ CI, 95\% confidence interval.

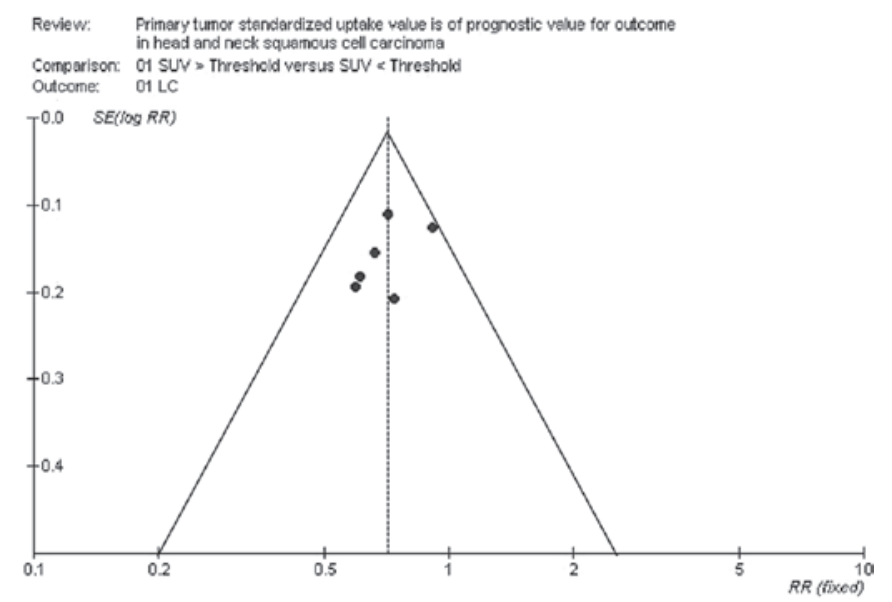

Figure 2. Funnel plot of included studies on local control. SUV, standardized uptake value; SE, standard error; RR, relative risk.

included patient characteristics (weight/height, glycaemia and histological subtype), ${ }^{18} \mathrm{~F}-\mathrm{FDG}$-PET acquisition protocol characteristics (injected dose of ${ }^{18} \mathrm{~F}-\mathrm{FDG}$, delay between injection and data acquisition and fasting duration) and technical parameters (investigation area, delay between head and neck CT and PET acquisition, SUV formula, type of PET engine, duration of emission time, duration of transmission time, attenuation and reconstruction parameters and type of SUV). The clinical and PET reports were scored on 21 and 14 points, respectively. A value between 0 and 2 was attributed to each item. The scores were expressed in percentage of the maximal theoretical value that can be obtained. When the results of a particular study were reported in more than one publication, only the most recent and complete data were included in the meta-analysis.

Statistical analysis. Data were extracted by Zhang and Nie and discrepancies were discussed and resolved by Dong. Data were entered into the Cochrane Collaboration Review Manager program RevMan 4.2.2. We measured the impact of SUV by relative risk (RR) between the local control distributions of two groups. For each trial, this RR was estimated by a method depending on the results provided in the publication. The most accurate method was through determining the total number of events and the number of patients at risk in each group, allowing calculation of the RR estimation. If the only exploitable data were in the form of graphical representations of local control distribution, they were used to extract the corresponding rates at specified times to reconstruct the RR estimate and its variance, with the hypothesis that the rate of patients censored was constant during the study follow-up. The individual RR point estimates were combined following acceptation of the null hypothesis of the homogeneity of the treatment effect across the various trials, using the RevMan 4.2.2 software to obtain a global RR estimate of the treatment effect. The RR was calculated using the fixed-effects method. In case of significant heterogeneity $(\mathrm{P}<0.05)$, the random-effects method was applied. This impact of SUV on local control was considered as statistically significant if the $95 \%$ confidence interval (95\% CI) for the overall RR did not overlap 1. All reported P-values were two-tailed. Finally, funnel plot asymmetry was used to detect any publication bias in the meta-analysis.

\section{Results}

Study characteristics. A total of 7 articles (10-16) on SUV in HNSCC were retrieved. One study was excluded from the analysis due to patient duplication (16) and the remaining 6 studies, published between 2002 and 2009, were considered eligible for this review. The sites of primary tumor in the studies included the oral cavity, oropharynx, nasopharynx, hypopharynx, larynx and maxilla. The follow-up time for local control, disease-free survival and overall survival ranged between 2 and 82.8 months per study. The principal characteristics of the 6 studies eligible for the meta-analysis are described in Table I. For the present study, SUV referred to the primary tumor, except in 5 patients ( 3 with unknown primary tumors and 2 with T1-2 tumors), in whom only the lymph nodes exhibited increased uptake. Consequently, for these 5 patients, the SUV of the lymph node was used as reference for correlation with local control. The main SUV 
characteristics reported in the publications are described in Table II.

Study quality. The methodological quality of the studies was moderate. Overall, the median quality score was $64 \%$, ranging between 53 and $70 \%$. The respective median values for the clinical and PET reports were 62\% (range, 55-69\%) and 66\% (range, 50-82\%), respectively.

High FDG uptake is a marker of poor outcome in HNSCC. The combined RR from the 6 reports for local control was 0.72 (95\% CI: 0.63-0.81). The combined RR was calculated using the fixed-effects method. The combined RR confirmed that high FDG uptake on PET is a marker for poor outcome in primary HNSCC. The results are detailed in Fig. 1. The funnel plot revealed a symmetrical distribution, indicating no evidence of substantial publication bias. The results are detailed in Fig. 2.

\section{Discussion}

Over the last decade, FDG-PET has become an important tool used to stage patients with HNSCC. Furthermore, in vivo imaging of human tumors with FDG-PET is a clinical extension of classical studies on carbohydrate metabolism. It was demonstrated that a high rate of glycolysis contributes to tumor growth, which may be exploited for malignancy grading with PET (17). The specific goal of this study was to evaluate the potential of SUV as a prognostic marker. The present meta-analysis confirmed that increased SUV of the primary tumor is a poor prognostic factor in patients with HNSCC.

Under the balanced circumstances of glucose metabolism, ${ }^{18} \mathrm{~F}-\mathrm{FDG}$ is phosphorylated. The retention products of FDG metabolism are consistent with the amount of glucose consumed in the cell. Therefore, ${ }^{18} \mathrm{~F}-\mathrm{FDG}$ may reflect the utilization of glucose in vivo. SUV is a semi-quantitative index that shows the characteristics of the ${ }^{18} \mathrm{~F}-\mathrm{FDG}$ tracer uptake, hence approximating the glucose metabolic rate. Commonly, some factors affecting the SUV include outlining of the region of interest, focus size, system resolution, reconstruction algorithm, patient factors (body weight, lean body weight and body surface area), non-tumor uptake caused by activating and acquisition time following drug injection. The time between injection and PET data acquisition in the eligible articles ranged between 45 and $60 \mathrm{~min}$; that makes SUV closer to the glucose metabolic rate. However, SUV estimates suffer from poor reproducibility between centers, due to the lack of standardization of the acquisition and processing protocols for its assessment. In our research, this poor reproducibility was proven by the broad range of threshold values that have been used in the literature to distinguish between patients with low and high survival. In order for it to be used as a functional prognostic factor in routine practice, a single SUV threshold distinguishing between patients should be agreed on, or an optimization method, used to determine the threshold for each center, should be established. To set a unanimous threshold, most variable factors affecting the SUV estimations should be defused or at least controlled. Reducing the large variability currently affecting SUV estimates is likely to enhance the prognostic value of SUV. In our research, we did not take into consideration the variable conditions under which SUV was obtained, due to the poor quality scores of the PET reports. Despite variability in the abovementioned factors, we were able to demonstrate that SUV was correlated with patient local control. Indeed, our research design calculated a RR for each study center to control the deviation of data, based on the SUV threshold used in the corresponding study, which somehow cancelled the difference in threshold factors used in different centers. By doing so, we were able to ignore the difference in SUV values between different centers and demonstrated that SUV is certainly worth considering as a prognostic factor.

Literature-based meta-analyses have the advantage of including published trials immediately available for analysis, with results that can be readily checked. In our meta-analysis, some biases may have occured. Although the funnel plot did not indicate publication bias, bias cannot definitely be ruled out, due to the small number of the studies and the low power of the test used to detect publication bias. Certain studies were not included, as separate data for head and neck cancer patients could not be obtained. Furthermore, one limitation of the present study is the lack of data from multicenter and large-scale perspective studies. To avoid some of the biases of literature-based meta-analyses, we aim to confirm our results in a meta-analysis of individual patient data, incorporating unpublished trials and updating results.

In conclusion, this meta-analysis provided evidence regarding the potential value of FDG uptake, as measured by SUV, in predicting local control in head and neck carcinomas. We are currently planning a meta-analysis based on individual patient data that will potentially reduce biases associated with literature-based meta-analyses.

\section{Acknowledgements}

The authors are indebted to the authors of the primary studies.

\section{References}

1. Lydiatt WM, Shah JP and Hoffman HT; Head and Neck Sites Task Force. American Joint Committee on Cancer: AJCC stage groupings for head and neck cancer: should we look at alternatives? A report of the Head and Neck Sites Task Force. Head Neck 23: 607-612, 2001.

2. Osman I, Sherman E, Singh B, et al: Alteration of p53 pathway in squamous cell carcinoma of the head and neck: impact on treatment outcome in patients treated with larynx preservation intent. J Clin Oncol 20: 2980-2987, 2002.

3. Izzo JG, Papadimitrakopoulou VA, Liu DD, et al: Cyclin D1 genotype, response to biochemoprevention and progression rate to upper aerodigestive tract cancer. J Natl Cancer Inst 95: 198-205, 2003.

4. Ang KK, Berkey BA, Tu X, et al: Impact of epidermal growth factor receptor expression on survival and pattern of relapse in patients with advanced head and neck carcinoma. Cancer Res 62: 7350-7356, 2002.

5. Rohren EM, Turkington TG and Coleman RE: Clinical applications of PET in oncology. Radiology 231: 305-332, 2004.

6. Minn H, Clavo AC, Grenman R and Wahl RL: In vitro comparison of cell proliferation kinetics and uptake of tritiated fluorodeoxyglucose and L-methionine in squamous-cell carcinoma of the head and neck. J Nucl Med 36: 252-258, 1995.

7. Haberkorn U, Strauss LG, Reisser C, et al: Glucose uptake, perfusion, and cell proliferation in head and neck tumors: relation of positron emission tomography to flow cytometry. J Nucl Med 32: 1548-1555, 1991. 
8. Vansteenkiste JF, Stroobants SG, Dupont PJ, De Leyn PR, Verbeken EK, Deneffe GJ, Mortelmans LA and Demedts MG: Prognostic importance of the standardized uptake value on ${ }^{18} \mathrm{~F}$-fluoro-2-deoxy-glucose-positron emission tomography scan in non-small-cell lung cancer: an analysis of 125 cases. Leuven Lung Cancer Group. J Clin Oncol 17: 3201-3206, 1999.

9. Berghmans T, Dusart M, Paesmans M, et al; European Lung Cancer Working Party for the IASLC Lung Cancer Staging Project: Primary tumor standardized uptake value (SUVmax) measured on fluorodeoxyglucose positron emission tomography (FDG-PET) is of prognostic value for survival in non-small cell lung cancer (NSCLC): a systematic review and meta-analysis (MA) by the European Lung Cancer Working Party for the IASLC Lung Cancer Staging Project. J Thorac Oncol 3: 6-12, 2008.

10. Brun E, Kjellen E, Tennvall J, et al: FDG PET studies during treatment: prediction of therapy outcome in head and neck squamous cell carcinoma. Head Neck 24: 127-135, 2002.

11. Allal AS, Slosman DO, Kebdani T, Allaoua M, Lehmann W and Dulguerov P: Prediction of outcome in head-and-neck cancer patients using the standardized uptake value of $2-\left[{ }^{18} \mathrm{~F}\right]$ fluoro-2-deoxy-D-glucose. Int J Radiat Oncol Biol Phys 59: 1295-1300, 2004.

12. Kim SY, Roh JL, Kim MR, et al: Use of ${ }^{18}$ F-FDG PET for primary treatment strategy in patients with squamous cell carcinoma of the oropharynx. J Nucl Med 48: 752-757, 2007.
13. Roh JL, Pae KH, Choi SH, et al: 2-[18 $\mathrm{F}]-$ Fluoro-2-deoxy-Dglucose positron emission tomography as guidance for primary treatment in patients with advanced-stage resectable squamous cell carcinoma of the larynx and hypopharynx. Eur J Surg Oncol 33: 790-795, 2007.

14. Liao CT, Chang JT, Wang HM, et al: Pretreatment primary tumor SUVmax measured by FDG-PET and pathologic tumor depth predict for poor outcomes in patients with oral cavity squamous cell carcinoma and pathologically positive lymph nodes. Int J Radiat Oncol Biol Phys 73: 764-771, 2009.

15. Torizuka T, Tanizaki Y, Kanno T, Futatsubashi M, Naitou K, Ueda Y and Ouchi Y: Prognostic value of ${ }^{18}$ F-FDG PET in patients with head and neck squamous cell cancer. AJR Am J Roentgenol 192: W156-W160, 2009.

16. Allal AS, Dulguerov P, Allaoua M, Haenggeli CA, EI-Ghazi el A Lehmann W and Slosman DO: Standardized uptake value of $2-\left[{ }^{18} \mathrm{~F}\right]$ fluoro-2-deoxy-D-glucose in predicting outcome in head and neck carcinomas treated by radiotherapy with or without chemotherapy. J Clin Oncol 20: 1398-1404, 2002.

17. Burk D, Woods M and Hunter J: On the significance of glucolysis for cancer growth, with special reference to Morris rat hepatomas. J Natl Cancer Inst 38: 839-863, 1967. 